\title{
Trousseau's syndrome associated with pulmonary pleomorphic carcinoma exhibiting aggressive features: A case report
}

\author{
SATORU OKADA ${ }^{1}$, AYA MIYAGAWA-HAYASHINO ${ }^{2}$, JUN FUJINAMI ${ }^{3}$, \\ TOMOKI NISHIMURA ${ }^{1}$, NARUMI ISHIKAWA ${ }^{1}$, HIROAKI TSUNEZUKA ${ }^{1}$, \\ MASANORI SHIMOMURA ${ }^{1}$, JUNICHI SHIMADA ${ }^{1}$ and MASAYOSHI INOUE ${ }^{1}$ \\ ${ }^{1}$ Division of Thoracic Surgery, Department of Surgery; ${ }^{2}$ Division of Surgical Pathology; ${ }^{3}$ Department of Neurology, \\ Graduate School of Medical Science, Kyoto Prefectural University of Medicine, Kyoto 602-8566, Japan
}

Received March 6, 2019; Accepted September 17, 2019

DOI: $10.3892 / \mathrm{mco} .2019 .1947$

\begin{abstract}
Trousseau's syndrome is characterized as an unexpected, cancer-related thrombotic event, such as a cerebral infarction or a deep vein thrombosis/pulmonary embolism. We describe the first reported case of Trousseau's syndrome with pulmonary pleomorphic carcinoma and aggressive features. A 74 year-old man presenting with a pulmonary mass, which was identified as pleomorphic carcinoma with extensive lymph node involvement, in the left lower lobe, underwent a left lower lobectomy. Immunohistochemical analysis revealed that neoplastic cells exhibited an extensive expression of tissue factors with a mucin-producing adenocarcinoma component. Three months postoperatively, diffuse infiltration rapidly appeared in the left lung, which was identified as lymphangitic carcinomatosis via bronchoscopy. Prior to treatment for cancer recurrence, the patient presented with a left hemiplegia due to a cerebral infarction via multiple thromboses, with no evidence of atherosclerotic or cardiogenic thrombi. Elevated D-dimer and carbohydrate antigen 125 levels and the presence of a fibrin thrombus retrieved from the occluded vessel suggested Trousseau's syndrome as the etiology of the brain infarction. A hypercoagulable state associated with the aggressive recurrence of pulmonary pleomorphic carcinoma, accompanied by cancer cell production of mucin and tissue factors may be a potential mechanism for cancer-related thrombosis.
\end{abstract}

Correspondence to: Professor Masayoshi Inoue, Division of Thoracic Surgery, Department of Surgery, Graduate School of Medical Science, Kyoto Prefectural University of Medicine, 465 Kajii-cho, Kawaramachi-Hirokoji, Kamigyo-ku, Kyoto 602-8566, Japan E-mail: mainoue@koto.kpu-m.ac.jp

Key words: Trousseau's syndrome, cerebral infarction, pulmonary pleomorphic carcinoma, mucin, tissue factor

\section{Introduction}

Armand Trousseau first reported Trousseau's syndrome, which is characterized as a cerebral embolism due to hypercoagulability resulting from malignant tumors, in 1865 (1). Trousseau's syndrome is now defined as an unexpected cancer-related thrombotic event, such as a cerebral infarction or deep vein thrombosis/pulmonary embolism (2). Trousseau's syndrome is recognized to be a fatal condition with a poor prognosis (3).

Cancer patients have an increased risk of arterial thromboembolism even before cancer diagnosis, and its risk is greater in more advanced stages of cancer $(4,5)$. Among patients with various cancer types, patients with lung cancer had the greatest excess risk of arterial thromboembolism, with a 3 month cumulative incidence of $6.5 \%$ since diagnosis (compared with $1.2 \%$ in control patients) (4). The precise mechanism underlying Trousseau's syndrome is not yet known. However, the clear correlation between cancer stage and arterial thromboembolism risk suggests a biological gradient between cancer activity and arterial thromboembolism risk (4). Circulating micro-particles, secretion of pro-coagulant factors, and alterations in platelet activity and endothelial function are potential relevant factors of Trousseau's syndrome $(2,4)$.

There have been several reports on lung adenocarcinoma accompanied with Trousseau's syndrome (6-9). Here, we present a rare case of pulmonary pleomorphic carcinoma accompanied with Trousseau's syndrome that manifested as thromboembolic brain infarction at the time of postoperative cancer recurrence.

\section{Case report}

A 74 year-old asymptomatic man was referred to our department for the evaluation of a radiographically identified solitary pulmonary mass. He was a current heavy smoker (38 pack-years). Chest computed tomography (CT) revealed a well-circumscribed peripheral mass (diameter: $38 \mathrm{~mm}$ ) in the lower lobe of the left lung (Fig. 1A). A honeycomb finding was also detected in the dorsal portion of the bilateral lung, indicating the presence of interstitial pneumonia. A fluorine-18-fluorodeoxyglucose (FDG) positron-emission tomography $\mathrm{CT}$ scan showed a strong 
accumulation of FDG (maximal level of standardized uptake value: 24.7$)$ in the mass. Serum carcinoembryonic antigen (CEA) and D-dimer levels were $16.0 \mathrm{ng} / \mathrm{ml}$ and $0.6 \mu \mathrm{g} / \mathrm{ml}$, respectively. Although transbronchial biopsy failed to yield a pathological diagnosis, the mass was suspected to be malignant; therefore, a left lower lobectomy with systemic mediastinal lymph node dissection was performed under video-assisted thoracoscopic surgery.

Lung specimens were fixed in $10 \%$ buffered formalin overnight at room temperature and embedded in paraffin. The paraffin-embedded specimens were sliced $4 \mu \mathrm{m}$ thick and stained with hematoxylin and eosin, Periodic acid Schiff (PAS), and PAS with diastase. Immunohistochemistry was performed using an autoimmunostainer according to manufacturer instructions (Ventana XT System Benchmark; Ventana Medical System, Inc.). The microscopic analyses were performed by a Nikon Eclipse $\mathrm{Ci}$ microscope and DS-Fi2 camera (Nikon Instruments). Pathological examination revealed pleomorphic carcinoma, measuring $3.5 \mathrm{~cm}$ at its greatest diameter, with visceral pleural and significant lymphatic invasion. Separate tumor nodules were present in the same lobe. The bronchial stump was tumor positive. The tumor extensively involved mediastinal lymph nodes and was graded as pT3N2M0, pStage IIIB. The tumor mainly comprised giant cells with high-grade pleomorphism and abnormal mitosis (approximately $60 \%$ of the tumor) (Fig. 2A), admixed with a solid adenocarcinoma component and papillary growth pattern at the periphery, which was positive for thyroid transcription factor-1 (clone 8G7G3/1, 1:200; DakoCytomation) (Fig. 2B). High expression of programmed death ligand-1 was confirmed in the tumor cells (clone 22C3 PharmDx, 1:100, tumor proportion score of 95\%; Agilent) (Fig. 2C). Meanwhile, the tumor was negative for epidermal growth factor receptor $(E G F R)$ mutations and anaplastic lymphoma kinase $(A L K)$ protein expression. The adenocarcinoma component was positive for periodic acid-Schiff (PAS) stain and resistant to diastase, suggesting mucin production (Fig. 2D). Moreover, most of the tumor cells were strongly positive for tissue factor (clone TF (H-9), 1:100; Santa Cruz Biotechnology, Inc.) (Fig. 2E).

Three months postoperatively, diffuse infiltration rapidly appeared in plain chest radiographs of the left lung (Fig. 1B), which was identified as lymphangitic carcinomatosis rather than an acute exacerbation of interstitial pneumonia, via bronchoscopy (Fig. 1C). Five days after examination and prior to treatment for cancer recurrence, the patient suddenly presented with dysarthria and left hemiplegia. Magnetic resonance imaging revealed acute ischemic stroke in the right hemisphere accompanied with subacute small infarcts in the left hemisphere and bilateral cerebellum (Fig. 3A). Magnetic resonance angiography revealed a right middle cerebral artery M2 segment occlusion (Fig. 3B). An echography and a chest $\mathrm{CT}$ showed no evidence of atherosclerotic thrombus or cardiac thrombus in the left atrium or in the stump of the resected pulmonary vein. Plasma D-dimer and fibrin/fibrinogen degradation product (FDP) levels were elevated at $17.6 \mu \mathrm{g} / \mathrm{ml}$ (normal <1.0) and $91.1 \mu \mathrm{g} / \mathrm{ml}$ (normal <5.0), as were the CEA and carbohydrate antigen 125 (CA125) levels $[73.4 \mathrm{ng} / \mathrm{ml}$ (normal <5.0) and $331 \mathrm{U} / \mathrm{ml}$ (normal <35)], respectively. Although fibrinogen level was slightly decreased to
$113 \mathrm{mg} / \mathrm{dl}$, the platelet count, prothrombin time international normalized ratio (PT-INR), APTT, and, antithrombin level were within normal limits $\left(14.7 \times 10^{4} / \mu 1,1.06,27.6 \mathrm{sec}\right.$, and $85 \%$, respectively), indicating that disseminated intravascular coagulation was not overt (10). He underwent mechanical thrombectomy with a stent retriever, and partial recanalization was achieved. However, intracerebral hemorrhage was observed after endovascular treatment. The pathology of the retrieved thrombus showed that almost all parts consisted of fibrin without red blood cells (Fig. 2F). These findings and pathological findings of the primary lung cancer suggested Trousseau's syndrome as the etiology of the cerebral infarction. Five days following the onset of cerebral infarction, our patient died of an intracerebral hemorrhage.

\section{Discussion}

Cancer-related arterial thromboembolism is often accompanied with lung, pancreatic, gastric, and colon cancer (4). The histology of most reported cases of lung cancer with Trousseau's syndrome involves adenocarcinoma (6-9). To our knowledge, this is the first reported case of Trousseau's syndrome due to pulmonary pleomorphic carcinoma.

Potential causes of brain infarction that occur following lung cancer surgery include atherosclerotic thrombus, cardiogenic thrombus, thrombus from the stump of the resected pulmonary vein, tumor embolization, and Trousseau's syndrome $(2,11-13)$. In this case, elevated D-dimer levels and extensive production of tissue factor in the tumor cells indicated a hypercoagulable state at the time of lung cancer recurrence. Moreover, elevated CA125 levels, the presence of mucin-producing adenocarcinoma as a component of the pleomorphic carcinoma, and fibrin thrombus indicated the possibility of a cancer-related thrombus as an etiology of the brain infarction $(6,14,15)$. These findings suggested that Trousseau's syndrome in relation to recurrent pulmonary pleomorphic carcinoma was the most probable etiology.

Pulmonary pleomorphic carcinoma is a rare malignant lung tumor and exhibits poor prognosis. Pulmonary pleomorphic carcinoma is defined as a poorly differentiated non-small cell carcinoma (NSCC), namely, a squamous cell carcinoma, adenocarcinoma, or undifferentiated NSCC that contains at least $10 \%$ spindle and/or giant cells, or a carcinoma consisting only of spindle and giant cells (16). In our case, the pleomorphic carcinoma included mucin-producing solid adenocarcinoma that was revealed through PAS reaction, although hematoxylin and eosin staining failed to confirm this.

Cancer-producing mucins are large, heavily glycosylated molecules that can act as ligands for selectins, particularly L-selectin of leukocytes and P-selectin of endothelium and/or platelets, causing platelet aggregation without the help of thrombin (17). Mucins are considered to be a coagulation trigger, resulting in the induction of disseminated thrombosis (6). In this case, the elevated CA125 level, a potential biomarker for a mucin-producing tumor (18), was consistent with the presence of mucin in the tumor.

Tissue factor is a primary cellular initiator of fluid-phase blood coagulation that changes factor VII (FVII) to its activated form (FVIIa) and initiates the extrinsic coagulation pathway (2). Tumor cells of various malignancies sometimes 

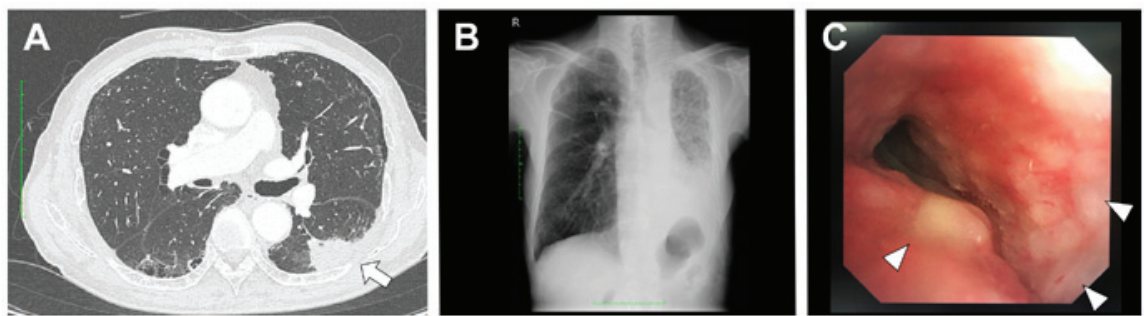

Figure 1. (A) Chest CT showing a 38-mm well-circumscribed mass in the lower lobe of the left lung (arrow). (B) Plain chest radiograph showing diffuse infiltration in the left lung. (C) Bronchoscopy showing intrabronchial diffuse recurrent tumor reflecting lymphangitic carcinomatosis (arrowheads). CT, computed tomography.
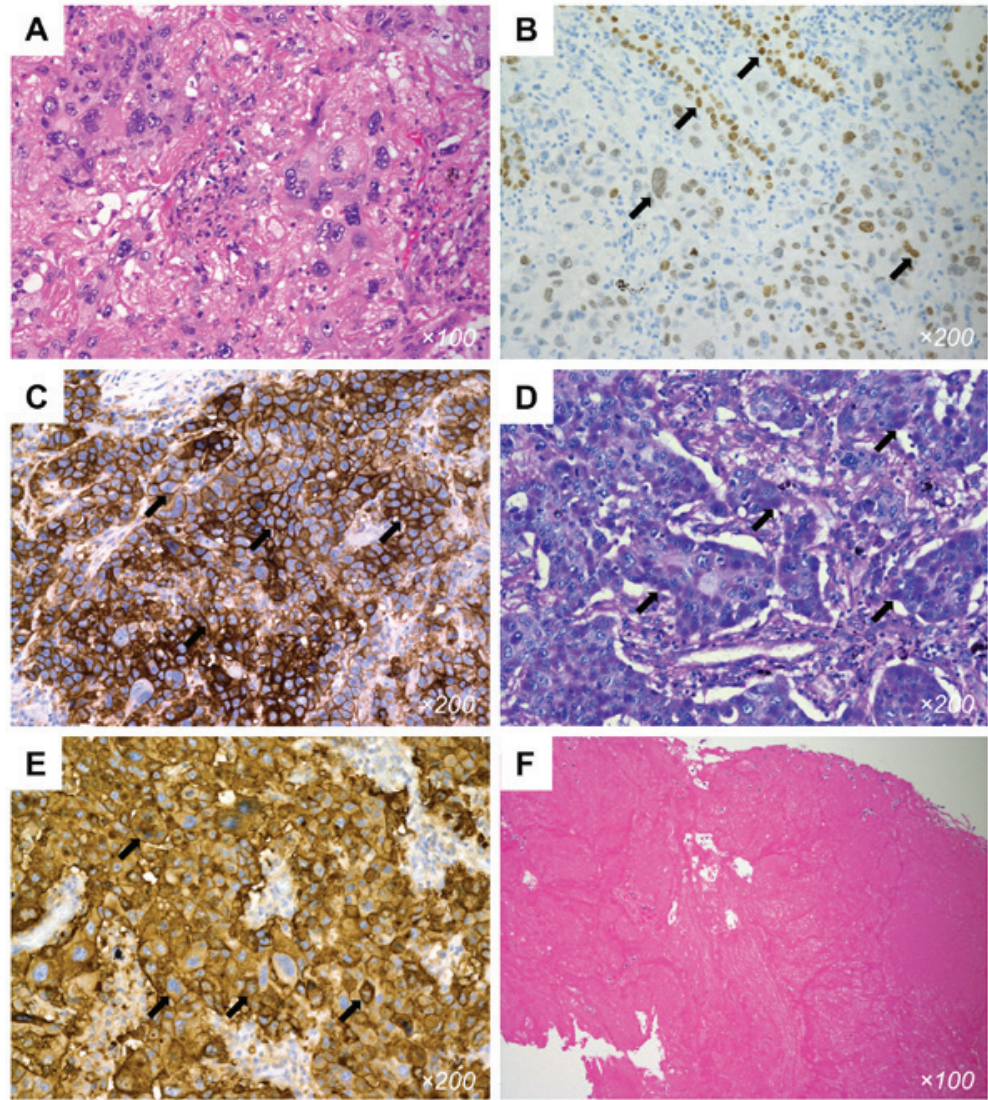

Figure 2. Histological examination of a tumor specimen. (A) Most of the tumor contained giant cells with atypia as pulmonary pleomorphic carcinoma (H\&E; magnification, x100). (B) Several tumor cell nests immunohistochemically positive for TTF-1 were identified (arrows), suggesting adenocarcinoma (TTF-1; magnification, x200). (C) Immunohistochemical staining revealed PD-L1 expression on 95\% of the tumor cells (arrows; PD-L1, clone 22C3; magnification, x200). (D) The tumor consisted, in part, of solid adenocarcinoma harboring PAS-positive intracytoplasmic mucin (arrows; PAS; magnification, x200). (E) Tumor cells were strongly positive for tissue factor [arrows; tissue factor, clone TF (H-9); magnification, x200]. (F) Retrieved thrombi from an occluded intracerebral vessel was composed of fibrin (H\&E; magnification, x100). H\&E, hematoxylin and eosin; PAS, periodic acid-Schiff stain; PD-L1, programmed death ligand-1; TTF-1, thyroid transcription factor-1.
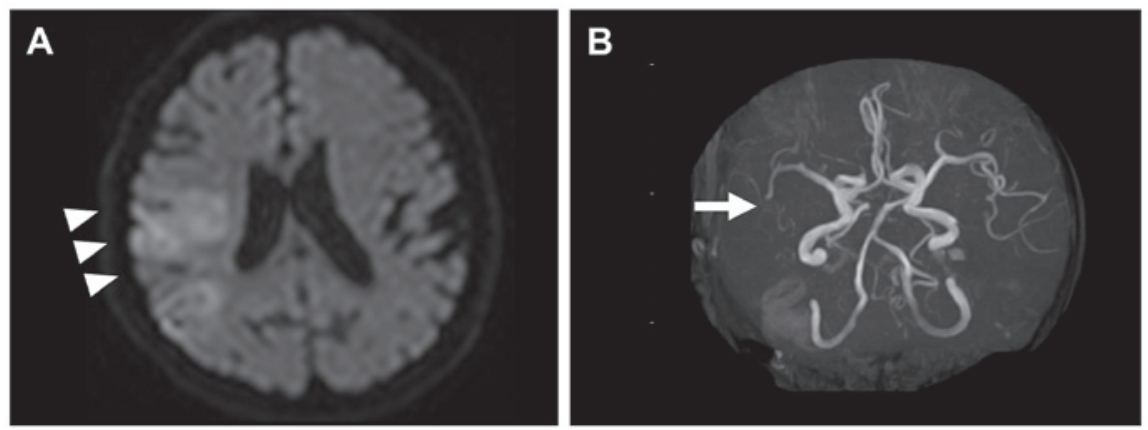

Figure 3. (A) Diffusion weighted magnetic resonance imaging of the brain showing acute cerebral infarction in the area concerning the right middle cerebral artery (arrowheads). (B) Magnetic resonance angiography showing a right middle cerebral artery M2 segment occlusion (arrow). 
produce tissue factor and may play an important role in the development of a thromboembolism in cancer patients $(7,19)$. Sato et al (7), reported that Trousseau's syndrome associated with tissue factor was produced by pulmonary adenocarcinoma. Sawada et al (19), reported that high tissue factor expression of tumor cells had close associations with advanced stage tumors and tumor recurrence, and approximately one-third of metastatic lung cancers had a strong tissue factor expression. Our recurrent case had aggressive features and expressed tissue factor extensively, which was consistent with the findings of a previous report. An increase in tissue factor production might partially explain the higher incidence of Trousseau's syndrome in patients with advanced stage malignancies.

Prognosis of cancer patients with stroke is poor (median survival 4.5 months), and $25 \%$ of them die within 30 days after the diagnosis of stroke (3). However, recently, a combination therapy comprising anti-coagulation treatment and chemotherapy or molecular targeted treatment has succeeded in inhibiting repeated thrombosis and controlling the tumor $(8,9)$. Pulmonary pleomorphic carcinoma often relates with high PD-L1 expression (20), and anti-PD-1/PD-L1 antibody treatment may be a promising treatment option (21). Early and accurate clarification of the etiology of a brain infarction may improve outcomes, especially for cases involving Trousseau's syndrome. Therefore, we recommend clinicians should not exclude the possibility of Trousseau's syndrome, even in diagnosed cases of pulmonary pleomorphic carcinoma.

In conclusion, we report the first case of Trousseau's syndrome accompanied with pulmonary pleomorphic carcinoma showing aggressive features and a hypercoagulable state at the time of recurrence. The mucin-producing component or tissue factor present in the tumor may be associated with a hypercoagulable state leading to cancer-related thrombosis. Monitoring of D-dimer and CA125 levels during postoperative follow-up after lung cancer surgery, especially in cases of aggressive recurrence patterns, could help early detection and treatment of a life-threatening cancer-related thrombosis.

\section{Acknowledgements}

Not applicable.

\section{Funding}

No funding was received.

\section{Availability of data and materials}

The datasets used and/or analysed during the present study are available from the corresponding author on reasonable request.

\section{Authors' contributions}

SO and MI conceived and designed the study. SO, JF, NI and $\mathrm{TN}$ collected the clinical data during the case study. AMH performed the pathological analyses. SO, JF and AMH wrote the manuscript. SO, AMH, JF, NI, TN, HT, MS, JS and MI interpreted the data and revised the clinical content. All authors read and approved the final manuscript of the manuscript, and agreed to be accountable for all aspects of this work.

\section{Ethics approval and consent to participate}

Not applicable.

\section{Patient consent for publication}

Written informed consent was obtained from the patient's kin for the publication of this data.

\section{Competing interests}

The authors declare that they have no competing interests.

\section{References}

1. Trousseau A: Plegmasia alba dolens. In: Lectures on clinical medicine, delivered at the Hotel-Dieu, Paris. Vol 5. The New Sydenham Society, London, pp281-332, 1865.

2. Varki A: Trousseau's syndrome: Multiple definitions and multiple mechanisms. Blood 110: 1723-1729, 2007.

3. Cestari DM, Weine DM, Panageas KS, Segal AZ and DeAngelis LM: Stroke in patients with cancer: Incidence and etiology. Neurology 62: 2025-2030, 2004.

4. Navi BB, Reiner AS, Kamel H, Iadecola C, Okin PM, Elkind MSV, Panageas KS and DeAngelis LM: Risk of arterial thromboembolism in patients with cancer. J Am Coll Cardiol 70: 926-938, 2017

5. Navi BB, Reiner AS, Kamel H, Iadecola C, Okin PM, Tagawa ST, Panageas KS and DeAngelis LM: Arterial thromboembolic events preceding the diagnosis of cancer in older persons. Blood 133: 781-789, 2019

6. Tachihara M, Nikaido T, Wang X, Sato Y, Ishii T, Saito K, Sekine S, Tanino Y, Ishida T and Munakata M: Four cases of trousseau's syndrome associated with lung adenocarcinoma. Intern Med 51: 1099-1102, 2012.

7. Sato T, Tsujino I, Ikeda D, Ieko M and Nishimura M: Trousseau's syndrome associated with tissue factor produced by pulmonary adenocarcinoma. Thorax 61: 1009-1010, 2006.

8. Masubuchi H, Maeno T, Uchida M, Kono S, Suzuki M, Takemura M, Yamaguchi A, Yamaguchi K, Kanbe M and Kitahara S: A case of trousseau syndrome caused by pulmonary adenocarcinoma that was controlled for one year and 10 months with thrombosis treatment using an EGFR tyrosine kinase inhibitor and chemotherapy. Respir Med Case Rep 15: 101-105, 2015.

9. Nonagase Y, Takeda M, Tanaka K, Hayashi H, Iwasa T and Nakagawa K: Treatment of EGFR mutation-positive non-small cell lung cancer complicated by Trousseau syndrome with gefitinib followed by osimertinib: A case report. Oncotarget 9: 29532-29535, 2018.

10. Asakura H, Takahashi H, Uchiyama T, Eguchi Y, Okamoto K, Kawasugi K, Madoiwa S and Wada H: Proposal for new diagnostic criteria for DIC from the Japanese society on thrombosis and hemostasis. Thromb J 14: 42, 2016.

11. Zhang YY, Cordato D, Shen Q, Sheng AZ, Hung WT and Chan DK: Risk factor, pattern, etiology and outcome in ischemic stroke patients with cancer: A nested case-control study. Cerebrovasc Dis 23: 181-187, 2007.

12. Hattori A, Takamochi K, Kitamura Y, Matsunaga T, Suzuki K, Oh S and Suzuki K: Risk factor analysis of cerebral infarction and clinicopathological characteristics of left upper pulmonary vein stump thrombus after lobectomy. Gen Thorac Cardiovasc Surg 67: 247-253, 2018.

13. Cho Y, Hida Y, Kaga K, Kato H, Iizuka M and Kondo S: Brain metastases secondary to tumor emboli from primary lung cancer during lobectomy. Ann Thorac Surg 86: 312-313, 2008.

14. Matsumoto N, Fukuda H, Handa A, Kawasaki T, Kurosaki Y, Chin M and Yamagata S: Histological examination of trousseau syndrome-related thrombus retrieved through acute endovascular thrombectomy: Report of 2 Cases. J Stroke Cerebrovasc Dis 25: e227-e230, 2016.

15. Ishikawa M, Nakayama K, Ishibashi T, Sato E, Nakamura K, Katagiri $\mathrm{H}$ and Kyo S: Case series of cerebral infarction with trousseau's syndrome associated with malignant gynecological tumors. Mol Clin Oncol 5: 138-142, 2016. 
16. Travis WD, Brambilla E, Nicholson AG, Yatabe Y, Austin JHM Beasley MB, Chirieac LR, Dacic S, Duhig E, Flieder DB, et al: The 2015 world health organization classification of lung tumors: Impact of genetic, clinical and radiologic advances since the 2004 classification. J Thorac Oncol 10: 1243-1260, 2015.

17. Wahrenbrock M, Borsig L, Le D, Varki N and Varki A: Selectin-mucin interactions as a probable molecular explanation for the association of trousseau syndrome with mucinous adenocarcinomas. J Clin Invest 112: 853-862, 2003.

18. Jovin TG, Boosupalli V,Zivkovic SA, Wechsler LR and Gebel JM High titers of CA-125 may be associated with recurrent ischemic strokes in patients with cancer. Neurology 64: 1944-1945, 2005.

19. Sawada M, Miyake S, Ohdama S, Matsubara O, Masuda S, Yakumaru K and Yoshizawa Y: Expression of tissue factor in non-small-cell lung cancers and its relationship to metastasis. Br J Cancer 79: 472-477, 1999.
20. Kim S, Kim MY, Koh J, Go H, Lee DS, Jeon YK and Chung DH: Programmed death-1 ligand 1 and 2 are highly expressed in pleomorphic carcinomas of the lung: Comparison of sarcomatous and carcinomatous areas. Eur J Cancer 51: 2698-2707, 2015.

21. Ikematsu Y, Yoneshima Y, Ijichi K, Tanaka K, Harada T, Oda Y, Nakanishi Y and Okamoto I: Marked response to pembrolizumab in a patient with pulmonary pleomorphic carcinoma highly positive for PD-L1. Lung Cancer 112: 230-231, 2017.

(i) (3) This work is licensed under a Creative Commons Attribution-NonCommercial-NoDerivatives 4.0 International (CC BY-NC-ND 4.0) License. 